\title{
An Example of Ill-Conditioning in the Numerical Solution of Singular Perturbation Problems*
}

\author{
By Fred W. Dorr
}

\begin{abstract}
The use of finite-difference methods is considered for solving a singular perturbation problem for a linear ordinary differential equation with an interior turning point. Computational results demonstrate that such problems can lead to very ill-conditioned matrix equations.
\end{abstract}

1. Introduction. Greenspan [8] has treated the following form of the steadystate Navier-Stokes equations:

$$
\begin{array}{ll}
\psi_{x x}+\psi_{y y}=-\omega & \text { in } G, \\
\omega_{x x}+\omega_{y y}+R\left(\psi_{x} \omega_{y}-\psi_{y} \omega_{x}\right)=0 & \text { in } G,
\end{array}
$$

where $G=(0,1) \times(0,1)$ and $R$ is the Reynolds number. We are interested in the asymptotic behavior of $\psi(x, y)=\psi(x, y ; R)$ and $\omega(x, y)=\omega(x, y ; R)$ as $R \rightarrow+\infty$. We impose the Dirichlet boundary conditions

$$
\begin{array}{ll}
\psi=0 & \text { on } \partial G, \\
\omega=1 & \text { on } \partial G \cap\{(x, y) \mid x=0 \text { or } y=1\}, \\
\omega=-1 & \text { on } \partial G \cap\{(x, y) \mid x=1 \text { or } y=0\} .
\end{array}
$$

In this case, we can extend the solution functions $\psi(x, y)$ and $\omega(x, y)$ across the line $x=y$ by skew symmetry. Thus we let $T=\{(x, y) \mid 0<x<y<1\}$, and we consider the problem

$$
\begin{array}{ll}
\psi_{x x}+\psi_{y y}=-\omega & \text { in } T, \\
\omega_{x x}+\omega_{y y}+R\left(\psi_{x} \omega_{y}-\psi_{y} \omega_{x}\right)=0 & \text { in } T, \\
\psi=0 \quad \text { on } \partial T, & \\
\omega=1 \quad \text { on } \partial T \cap\{(x, y) \mid x=0 \text { or } y=1\}, \\
\omega=0 \quad \text { on } \partial T \cap\{(x, y) \mid x=y\} .
\end{array}
$$

Dorr and Parter [5], [6] have considered the following one-dimensional analog of Eq. (1):

$$
\begin{array}{cc}
u^{\prime \prime}(t)=-v(t) & (0<t<1), \\
\epsilon v^{\prime \prime}(t)+u^{\prime}(t) v^{\prime}(t)=0 & (0<t<1), \\
u(0)=u(1)=0, \quad v(0)=v_{0}, \quad v(1)=v_{1},
\end{array}
$$

Received June 5, 1970, revised December 14, 1970.

AMS 1969 subject classifications. Primary 6562, 6570.

Key words and phrases. Ordinary differential equations, boundary-value problems, singular perturbation problems, finite-difference equations, matrix equations, ill-conditioning.

*This research was supported by the U. S. Atomic Energy Commission under Contract No. W-7405-ENG-36. 
where $\epsilon=1 / R>0$ and $0 \leqq v_{0}<v_{1}$. For this particular example, we have [5, Theorem 6]:

$$
\begin{aligned}
& \lim _{\epsilon \rightarrow 0+} u(t, \epsilon)=\frac{1}{4}\left(v_{0}+v_{1}\right)\left(t-t^{2}\right) \quad(0 \leqq t \leqq 1), \\
& \lim _{\epsilon \rightarrow 0+} v(t, \epsilon)=\frac{1}{2}\left(v_{0}+v_{1}\right) \quad(0<t<1) .
\end{aligned}
$$

We remark that $u^{\prime}(t, \epsilon)$ has exactly one interior zero for $\epsilon>0$, so that Eq. (2) represents a singular perturbation problem with an interior turning point.

The procedure used by Greenspan to generate approximate solutions to Eq. (1) is to define a uniform grid of mesh points on $T$ with mesh width $h>0$. He replaces the differential equations by finite-difference equations, and examines the behavior of these discrete solutions as $R \rightarrow+\infty$ with $h>0$ held fixed. The corresponding finitedifference results for the one-dimensional model, Eq. (2), are given in [3], [4].

In this paper, we examine in detail the linear problem

$$
\begin{gathered}
\epsilon w^{\prime \prime}(t)+\left(\frac{1}{2}-t\right) w^{\prime}(t)=0 \quad(0<t<1), \\
w(0)=v_{0}, \quad w(1)=v_{1} .
\end{gathered}
$$

The asymptotic behavior of $w(t, \epsilon)$ is the same as that of $v(t, \epsilon)$,

$$
\lim _{\epsilon \rightarrow 0+} w(t, \epsilon)=\frac{1}{2}\left(v_{0}+v_{1}\right) \quad(0<t<1) .
$$

To study the solution of Eq. (3) by finite-difference methods, we define a uniform grid of mesh points on the interval $[0,1]$ with mesh width $h>0$. The differential equation is replaced by a difference equation, and we study the behavior of the solution to the difference equation as $\epsilon \rightarrow 0+$ with $h>0$ held fixed. We show in Section 3 that the solutions to the differential equation and the difference equation have the same asymptotic behavior when the difference equation is defined in the proper way. We also give in Section 4 some numerical results which show that the matrix equation corresponding to the difference equation can be very ill-conditioned.

A problem similar to Eq. (3) is the following:

$$
\begin{gathered}
\epsilon y^{\prime \prime}(t)-\left(\frac{1}{2}-t\right) y^{\prime}(t)=0 \quad(0<t<1), \\
y(0)=v_{0}, \quad y(1)=v_{1},
\end{gathered}
$$

and then

$$
\begin{aligned}
\lim _{\epsilon \rightarrow 0+} y(t, \epsilon) & =v_{0} \quad \text { if } 0 \leqq t<\frac{1}{2}, \\
& =v_{1} \quad \text { if } \frac{1}{2}<t \leqq 1 .
\end{aligned}
$$

The analysis of the finite-difference equation can also be applied to this problem. However, the corresponding matrix equation is not ill-conditioned, and we will not consider this particular problem further. Some related computational results are given in [4].

There are two primary reasons for treating the simple linear example, Eq. (3). First, this choice greatly simplifies the presentation of the methods developed in [4] to determine the asymptotic behavior of the solutions to the nonlinear difference equations corresponding to Eq. (2). Second, we want to give some detailed com- 
putational results in this paper, and it is easier to discuss these results for the linear problem. Similar computations for the nonlinear problem are discussed in [3], and the same type of ill-conditioned behavior is apparent there.

It should be noted that we cannot obtain the precise behavior of the solution to Eq. (3) in the "boundary layers" near $t=0$ and $t=1$ by fixing $h>0$ and letting $\epsilon \rightarrow 0$. There are a number of other methods that can be used to determine the behavior in these boundary layers. For example, Pearson [11], [12] allows the position and number of mesh points to vary as $\epsilon \rightarrow 0$, and Murphy [9] considers initial-value problems with $h=\epsilon^{\alpha}$ for a fixed $\alpha>1$. We also mention the related work of Price and Varga [14] on the use of variational methods for problems with boundary layer behavior.

In Section 2 we introduce the notation and some preliminary results that will be used in the remainder of the paper. The asymptotic behavior of solutions to difference equations is treated in Section 3. Computational results for the linear problem are discussed in Section 4, and in Section 5 we give some special methods that can be used to successfully solve this particular problem.

2. Notation and Preliminary Results. We introduce a mesh size $h=1 /(N+1)$ (where $N$ is a positive integer), and mesh points $x_{i}=j h$. Define the following difference operators:

$$
\begin{aligned}
W_{x}(t) & =\left[\frac{W(t+h)-W(t)}{h}\right], \\
W_{x}(t) & =\left[\frac{W(t)-W(t-h)}{h}\right], \\
g(t) \frac{\partial W(t)}{\partial x} & =g(t) W_{x}(t) \text { if } g(t) \geqq 0, \\
& =g(t) W_{x}(t) \text { if } g(t)<0, \\
W_{\hat{x}}(t) & =\left[\frac{W(t+h)-W(t-h)}{2 h}\right] \\
W_{x x}(t) & =\left[\frac{W(t+h)-2 W(t)+W(t-h)}{h^{2}}\right] .
\end{aligned}
$$

If $W(t)$ is a smooth function and $g(t)$ is bounded, it is well known that $W_{x}(t)=$ $W^{\prime}(t)+O(h), W_{\hat{x}}(t)=W^{\prime}(t)+O(h), g(t) \partial W(t) / \partial x=g(t) W^{\prime}(t)+O(h), W_{\hat{x}}(t)=$ $W^{\prime}(t)+O\left(h^{2}\right)$, and $W_{x x}(t)=W^{\prime \prime}(t)+O\left(h^{2}\right)$.

Let $A$ be an $N \times N$ tridiagonal matrix of the form

$$
A=\left[\begin{array}{ccccccc}
b_{1} & c_{1} & & & & 0 & \\
a_{2} & b_{2} & c_{2} & & & & \\
& \cdot & \cdot & \cdot & & & \\
& & \cdot & \cdot & \cdot & & \\
& & & \cdot & \cdot & \cdot & \\
& 0 & & & a_{N-1} & b_{N-1} & c_{N-1} \\
& & & & & a_{N} & b_{N}
\end{array}\right] .
$$


We then write $A=\left[a_{i} b_{i} c_{i}\right]$. Let $L_{h}$ be a three-point difference operator of the form

$$
L_{h} W\left(x_{i}\right)=a_{i} W\left(x_{i-1}\right)+b_{i} W\left(x_{i}\right)+c_{i} W\left(x_{i+1}\right) .
$$

It is then easy to show that the boundary-value problem

$$
\begin{gathered}
L_{h} W\left(x_{i}\right)=f_{i} \quad(1 \leqq j \leqq N), \\
W(0)=f_{0}, \quad W(1)=f_{N+1},
\end{gathered}
$$

is equivalent to the matrix equation

$$
A \mathrm{~W}=\overline{\mathrm{f}},
$$

where

$$
\mathrm{W}=\left[\begin{array}{c}
W\left(x_{1}\right) \\
W\left(x_{2}\right) \\
\vdots \\
W\left(x_{N}\right)
\end{array}\right]
$$

and

$$
\begin{aligned}
\bar{f}_{i} & =f_{1}-a_{1} f_{0} & & \text { if } j=1, \\
& =f_{i} & & \text { if } 2 \leqq j \leqq N-1, \\
& =f_{N}-c_{N} f_{N+1} & & \text { if } j=N .
\end{aligned}
$$

We now prove two useful matrix results.

LEMMA 1. Let $A=\left(a_{i j}\right)$ be a lower Hessenberg matrix (i.e., $a_{i j}=0$ for $j \geqq i+2$ ). Assume that: (i) $a_{i, j+1} \neq 0(1 \leqq j \leqq N-1)$, and (ii) there exists a vector $\mathrm{W}$ such that $A \mathrm{~W}=\mathrm{e}_{N}$, where $\mathrm{e}_{N}$ is the Nth unit vector. Then $\operatorname{det} A \neq 0$.

Remark. A similar result holds for an upper Hessenberg matrix $A$ if we assume that: (i) $a_{j, j-1} \neq 0(2 \leqq j \leqq N)$, and (ii) there exists a vector $\mathrm{W}$ such that $A \mathrm{~W}=\mathrm{e}_{1}$.

Proof. Let $A^{T} \mathbf{Y}=0$. Then

$$
0=\mathrm{W}^{T} A^{T} \mathbf{Y}=\mathbf{e}_{N}^{T} \mathbf{Y}=Y_{N},
$$

and we can back-substitute in the equation $A^{T} \mathbf{Y}=0$ to find that $\mathbf{Y}=\mathbf{0}$.

LEMMA 2. Let $L_{h}$ be the difference operator defined in Eq. (4), and assume that:

$$
\begin{array}{ll}
\text { (i) } a_{i}+b_{i}+c_{i}=0 & (1 \leqq j \leqq N), \\
\text { (ii) } c_{i} \neq 0 & (1 \leqq j \leqq N) .
\end{array}
$$

Let $\Delta_{i}=\prod_{k=1}^{i}\left(a_{k} / c_{k}\right)$, and assume that $\left(1+\sum_{i=1}^{N} \Delta_{i}\right) \neq 0$.

Then there is a unique solution to the boundary-value problem given in Eq. (5). If $f_{i}=0$ for $1 \leqq j \leqq N$, the solution is given by

$$
W\left(x_{i}\right)=f_{0}+\left(f_{N+1}-f_{0}\right)\left(1+\sum_{i=1}^{i-1} \Delta_{i}\right)\left(1+\sum_{i=1}^{N} \Delta_{i}\right)^{-1} \quad(1 \leqq j \leqq N) .
$$

Proof. Let $A=\left[a_{i} b_{i} c_{i}\right]$ be the tridiagonal matrix corresponding to the operator $L_{h}$. Equation (7) can be used to exhibit a solution to the equation $A \mathrm{~W}=\mathrm{e}_{N}$, and the result then follows from Lemma 1. 
Remarks. (i) Under the conditions given in Eq. (6), it can be shown that

$$
\operatorname{det} A=(-1)^{N}\left(1+\sum_{i=1}^{N} \Delta_{i}\right) \prod_{i=1}^{N} c_{i},
$$

so that the proof of the nonsingularity of $A$ is immediate. However, we have stated the lemmas in this form because we need the representation in Eq. (7) later in the paper, and also because Lemma 1 is interesting in its own right. We also note that Eq. (8) can be used to show that the representation in Eq. (7) is actually Cramer's rule for the matrix $A$.

(ii) A result similar to Lemma 2 holds if we assume that $a_{i} \neq 0(1 \leqq j \leqq N)$ in Eq. (6), and also that $\left(1+\sum_{i=1}^{N} \Gamma_{i}\right) \neq 0$ where $\Gamma_{i}=\prod_{k=i}^{N}\left(c_{k} / a_{k}\right)(\mathrm{cf}$. [4, Lemma 3]).

3. Asymptotic Behavior of Solutions to Difference Equations. We now consider the linear boundary-value problem given in Eq. (3). The first difference equation treated is

$$
\begin{gathered}
\epsilon W_{x \dot{x}}\left(x_{i}\right)+\left(\frac{1}{2}-x_{i}\right) \frac{\partial W\left(x_{i}\right)}{\partial x}=0 \quad(1 \leqq j \leqq N), \\
W(0)=v_{0}, \quad W(1)=v_{1} .
\end{gathered}
$$

We examine the asymptotic behavior of $W\left(x_{i}, \epsilon\right)$ as $\epsilon \rightarrow 0+$ with $h>0$ held fixed.

THEOREM 1. For each $\epsilon>0$ there is a unique solution $W\left(x_{i}, \epsilon\right)$ to Eq. (9), and

$$
\lim _{\epsilon \rightarrow 0+} W\left(x_{i}, \epsilon\right)=\frac{1}{2}\left(v_{0}+v_{1}\right) \quad(1 \leqq j \leqq N) .
$$

The proof follows easily from Lemma 2. Note that the asymptotic behavior of $W\left(x_{i}, \epsilon\right)$ is the same as that of $w(t, \epsilon)$. If $L_{b}$ is the difference operator given by

$$
L_{h} W=\epsilon W_{x z}+\left(\frac{1}{2}-x_{i}\right) \frac{\partial W}{\partial x} \quad(\epsilon>0),
$$

then $L_{\mathrm{A}}$ is an operator of positive type (cf. [4]). This fact immediately implies that Eq. (9) has a unique solution, and in fact $W\left(x_{i}, \epsilon\right)$ is a monotone increasing function of $x_{i}$ for each fixed $\epsilon>0$. Since $w(t, \epsilon)$ also has this property, we see that $W\left(x_{i}, \epsilon\right)$ does indeed provide a qualitatively satisfactory approximation to $w(t, \epsilon)$.

As we remarked earlier, the difference operator $\partial W(t) / \partial x$ is a first-order approximation to $W^{\prime}(t)$ (i.e., $\partial W(t) / \partial x=W^{\prime}(t)+O(h)$ ), while $W_{\hat{x}}(t)$ is a second-order approximation to $W^{\prime}(t)$ (i.e., $W_{\hat{t}}(t)=W^{\prime}(t)+O\left(h^{2}\right)$ ). The next result shows that one should not always use a "more accurate" approximation to a differential operator. Rather, the choice of a difference operator must depend on the problem under consideration.

THEOREM 2. Consider the difference equation

$$
\begin{gathered}
\epsilon W_{x \hat{x}}\left(x_{i}\right)+\left(\frac{1}{2}-x_{j}\right) W_{\hat{x}}\left(x_{i}\right)=0 \quad(1 \leqq j \leqq N), \\
W(0)=v_{0}, \quad W(1)=v_{1} .
\end{gathered}
$$

Then there exists an $\epsilon_{0}=\epsilon_{0}(h)>0$ such that Eq. (10) has a unique solution $W\left(x_{i}, \epsilon\right)$ for $0<\epsilon \leqq \epsilon_{0}$. The asymptotic behavior is the following, where $M=[(N+1) / 2]$ : 
Case 1. $N$ even.

$$
\begin{aligned}
\lim _{\epsilon \rightarrow 0+} W\left(x_{i}, \epsilon\right) & =v_{0} \quad \text { if } j \text { is even, } \\
& =v_{1} \text { if } j \text { is odd. }
\end{aligned}
$$

Case 2. $N$ odd, $M$ odd.

$$
\begin{aligned}
& \lim _{\epsilon \rightarrow 0+} W\left(x_{i}, \epsilon\right)=\frac{1}{2}\left(v_{0}+v_{1}\right) \text { if } j \text { is odd, } \\
& =v_{0} \quad \text { if } j \text { is even, } 2 \leqq j \leqq M-1 \text {, } \\
& =v_{1} \quad \text { if } j \text { is even, } \quad M+1 \leqq j \leqq N-1 \text {. }
\end{aligned}
$$

Case 3. $N$ odd, $M$ even. If $j$ is odd,

$$
\begin{aligned}
\lim _{\epsilon \rightarrow 0+} W\left(x_{i}, \epsilon\right) & =+\infty \quad \text { if } 1 \leqq j \leqq M-1, \\
& =-\infty \quad \text { if } M+1 \leqq j \leqq N .
\end{aligned}
$$

If $j$ is even, we let

$$
\gamma_{i}=2 h \sum_{i=1}^{i / 2}\left(\frac{1}{2}-x_{2 i-1}\right)^{-1}
$$

and then

$$
\begin{aligned}
\lim _{c \rightarrow 0+} W\left(x_{i}, \epsilon\right) & =v_{0}+\left(v_{1}-v_{0}\right)\left(\frac{\gamma_{i}}{2 \gamma_{M}}\right) \quad \text { if } 2 \leqq j \leqq M \\
& =v_{1}-\left(v_{1}-v_{0}\right)\left(\frac{\gamma_{N+1-i}}{2 \gamma_{M}}\right) \quad \text { if } M+2 \leqq j \leqq N-1
\end{aligned}
$$

The proof of the theorem again follows from Lemma 2. Observe that for small values of $\epsilon$ the solution $W\left(x_{i}, \epsilon\right)$ to the centered difference equation, Eq. (10), is not monotone in $x_{i}$. It is possible to define a second-order approximation to $W^{\prime}(t)$ so that the solution to the corresponding difference equation is monotone (e.g., see [13, Section 3D. However, this approximation yields a four-point difference operator, and the techniques used in this section cannot be used to determine the asymptotic behavior of the solution to the difference equation.

4. Computational Results. We now consider the computational aspects of solving the difference equations. As we have seen in Section 3, it is essential to use the difference $\partial W / \partial x$ if we want to let $\epsilon \rightarrow 0+$ with $h>0$ held fixed. We therefore consider the particular problem

$$
\begin{gathered}
\epsilon W_{x i}\left(x_{i}\right)+\left(\frac{1}{2}-x_{i}\right) \frac{\partial W\left(x_{j}\right)}{\partial x}=0 \quad(1 \leqq j \leqq N), \\
W(0)=1, \quad W(1)=3 .
\end{gathered}
$$

This is equivalent to the matrix equation 


$$
A \mathrm{~W}=\left[\begin{array}{c}
-a_{1} \\
0 \\
\vdots \\
0 \\
-3 c_{N}
\end{array}\right] \equiv \mathrm{Y},
$$

where $A=A(\epsilon)=\left[a_{i} b_{i} c_{i}\right]$,

$$
\begin{aligned}
a_{i} & =-\frac{\epsilon}{h^{2}} & & \text { if } 1 \leqq j \leqq\left[\frac{N+1}{2}\right], \\
& =-\frac{\epsilon}{h^{2}}+\frac{1}{h}\left(\frac{1}{2}-x_{i}\right) & & \text { if }\left[\frac{N+1}{2}\right]+1 \leqq j \leqq N, \\
c_{i} & =-\frac{\epsilon}{h^{2}}-\frac{1}{h}\left(\frac{1}{2}-x_{i}\right) & & \text { if } 1 \leqq j \leqq\left[\frac{N+1}{2}\right], \\
& =-\frac{\epsilon}{h^{2}} & & \text { if }\left[\frac{N+1}{2}\right]+1 \leqq j \leqq N,
\end{aligned}
$$

and $b_{i}=-\left(a_{i}+c_{i}\right)$. Notice that the coefficients satisfy

$$
\begin{array}{ll}
a_{j} \leqq-\epsilon / h^{2}<0 & (1 \leqq j \leqq N), \\
c_{j} \leqq-\epsilon / h^{2}<0 & (1 \leqq j \leqq N) .
\end{array}
$$

We first list some relevant properties of the tridiagonal matrix $A$ :

(i) $A$ is irreducibly diagonally dominant, and hence nonsingular [15, p. 23]. Furthermore, $A$ is an $M$-matrix, and $A^{-1}>0[15$, p. 85].

(ii) $A$ is symmetrizable [16, pp. $335-336]$. That is, there is a positive definite diagonal matrix $D$ such that $D^{-1} A D$ is symmetric.

(iii) The eigenvalues of $A$ are positive, so that $D^{-1} A D$ is positive definite [15, p. 23].

(iv) The Gauss-Seidel and Jacobi iterative methods for solving $A \mathrm{~W}=\mathrm{Y}$ both converge [15, p. 84 and pp. 107-108]. Furthermore, if $\rho_{G S}\left[\rho_{J}\right]$ is the spectral radius of the Gauss-Seidel [Jacobi] iteration matrix, then $\rho_{G S}=\rho_{J}^{2}<1$.

Because of these properties, it would seem to be a relatively easy matter to solve the equation $A \mathrm{~W}=\mathrm{Y}$. In Table I we summarize some computational results for this problem with $N=100$ and four values of $\epsilon$. Although only the 50th component of $W\left(x_{i}, \epsilon\right)$ is given, the behavior is typical of the form of the solution. The methods of solution used are:

(1) Double-precision Gaussian elimination with no interchanges, in the form $W_{i}=d_{i}+\left(1+e_{i}\right) W_{i+1}$.

(2) Gaussian elimination with row interchanges.

(3) Gaussian elimination with no interchanges, in the form $W_{i}=d_{i}+e_{i} W_{i-1}$.

(4) Gaussian elimination with no interchanges, in the form $W_{i}=d_{i}+e_{i} W_{i+1}$.

(5) Gaussian elimination with no interchanges, in the form $W_{j}=d_{j}+\left(1+e_{j}\right) W_{i+1}$.

(6) A method due to Babuska [1, pp. 15-16],** which can be derived by combining methods (3) and (4).

** In a recent report [2], Babuška has described another variant of Gaussian elimination which he has successfully applied to this problem. 
TABLE I

Solutions to the Difference Equation

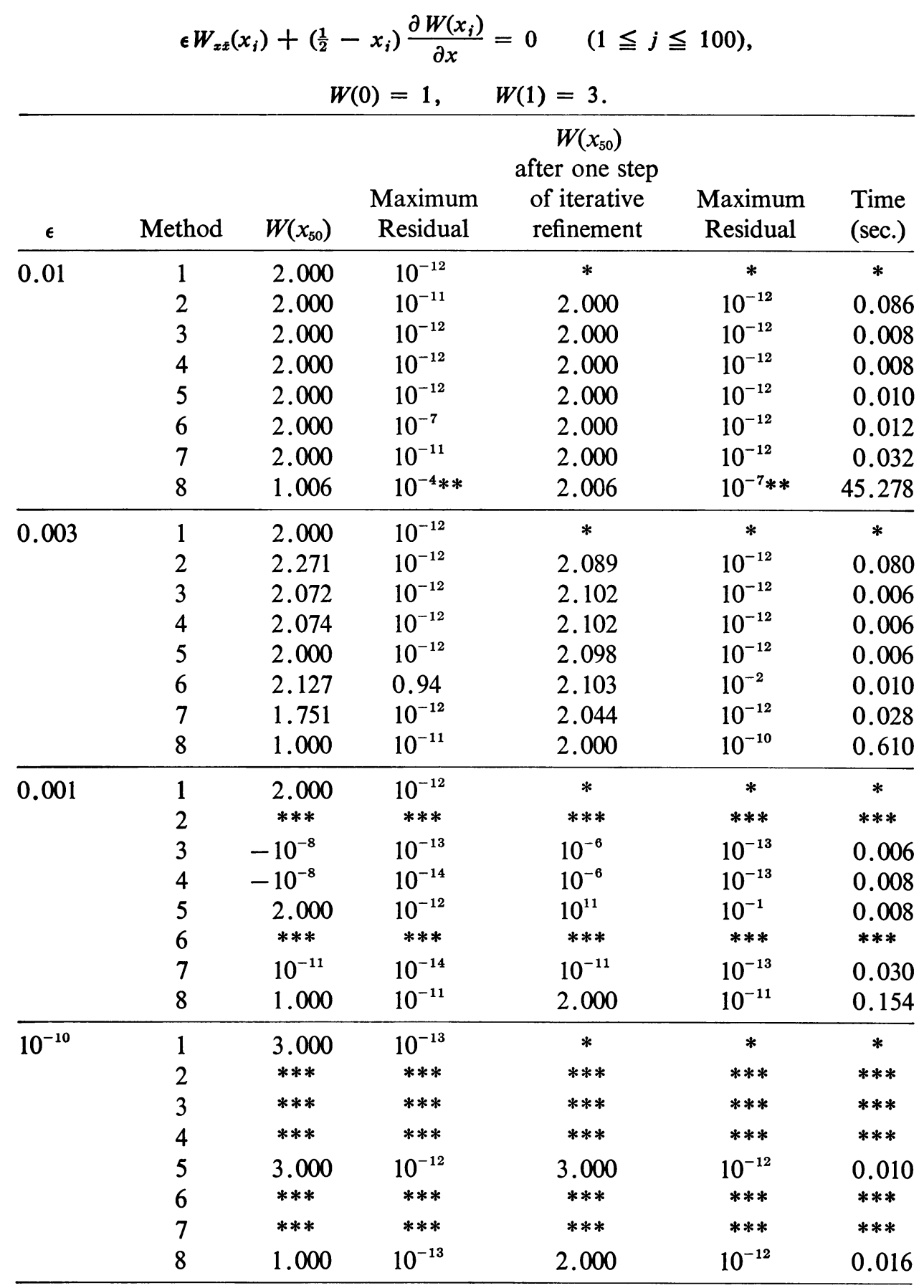

* Not applicable.

** Failed to converge in 10,000 iterations.

*** Method failed due to either overflow or underflow in arithmetic operations. 
(7) A method that symmetrizes $A$, and then uses Cholesky decomposition.

(8) Gauss-Seidel iteration with initial guess $W_{i}^{(0)} \equiv 1$. The criterion for convergence is that the maximum relative difference between successive iterates be less than $10^{-12}$.

We remark that all of the computations discussed in this paper were performed on a CDC 6600 computer.

A number of comments should be made about these numerical results:

(i) For $\epsilon=0.01$, the rate of convergence of the Gauss-Seidel method is very slow, which means that $\rho_{G S}$ is very close to 1 . For $\epsilon=0.001, \rho_{G S}$ has increased, and yet the rate of convergence appears to be much faster. In addition, for $\epsilon=0.001$ the application of Gauss-Seidel before the step of iterative refinement "converges" to the incorrect solution $W\left(x_{i}, \epsilon\right) \equiv 1$. The explanation behind this apparent contradiction is that the correction in each iteration of Gauss-Seidel is so small that the method appears to have converged, even though this is not the case. The significance of this example is to again demonstrate the well-known fact that one cannot always determine whether an iterative method has converged by simply looking at the difference between successive iterates.

(ii) For $\epsilon=0.003$, note that all of the methods (except (6)) give answers that look quite reasonable, but which are nevertheless incorrect. Also note that these erroneous solutions yield very small residuals.

(iii) For $\epsilon=0.001$, all of the single-precision methods show a marked degree of numerical instability. We remark that the double-precision method (1) also fails for $\epsilon$ small enough, as the result for $\epsilon=10^{-10}$ demonstrates.

These results indicate that the matrix $A(\epsilon)$ is becoming very ill-conditioned even for values of $\epsilon$ that are not very small (e.g., $\epsilon=0.003$ ). Let $0<\lambda_{1} \leqq \lambda_{2} \leqq \cdots \leqq \lambda_{N}$ be the eigenvalues of the difference matrix $A(\epsilon)$. For $\epsilon=0.003$ and $N=100$, we have calculated these eigenvalues numerically:

$$
\begin{aligned}
\lambda_{1}= & 1.8136 \times 10^{-12} \\
\lambda_{2}= & 1.0000 \\
\lambda_{3}= & 1.9326 \\
& \vdots \\
\lambda_{99}= & 199.8746 \\
\lambda_{100}= & 199.8746 .
\end{aligned}
$$

Thus we can calculate the approximate condition number

$$
\frac{\lambda_{100}}{\lambda_{1}}=1.1021 \times 10^{14}
$$

The single-precision word on the CDC 6600 computer corresponds to 14 or 15 decimal digits, which explains why we are having computational instability in the neighborhood of $\epsilon=0.003$.

It is interesting to note that preconditioning [7, pp. 9-10] does not significantly help in this problem. For $\epsilon=0.003$ and $N=100$, the eigenvalues of the precon- 
ditioned symmetrized difference matrix are

$$
\begin{aligned}
\lambda_{1}= & 2.7590 \times 10^{-14} \\
\lambda_{2}= & 1.4137 \times 10^{-2} \\
\lambda_{3}= & 2.6297 \times 10^{-2} \\
& \vdots \\
\lambda_{99}= & 1.9859 \\
\lambda_{100}= & 2.0000,
\end{aligned}
$$

and the approximate condition number is

$$
\frac{\lambda_{100}}{\lambda_{1}}=7.2491 \times 10^{13} \text {. }
$$

We have computed the spectral radius of the Gauss-Seidel iteration matrix for $\epsilon=0.003$ and $N=100$, and we have

$$
\rho_{G S}=1-\left(5.5179 \times 10^{-14}\right) .
$$

Thus the rate of convergence is extremely slow. The eigenvector corresponding to the largest eigenvalue is positive, so it cannot be orthogonal to the initial error (which is $e_{i}^{(0)} \equiv W_{i}-W_{i}^{(0)} \cong 2-1>0$ ).

In Table II we give some values of the smallest eigenvalue $\lambda_{1}(\epsilon)$ of the difference matrix $A(\epsilon)$ for various values of $\epsilon$. The values in the table are for a fixed $N=100$, but the results for other values of $N$ are similar. If $M=[(N+1) / 2]$, it can be shown that

$$
\begin{aligned}
\operatorname{det}(A(0)-\lambda I) & =\lambda(\lambda-1) \prod_{i=1}^{M-1}\left(\lambda-\frac{2 j+1}{2}\right)^{2} & & \text { if } N \text { is even, } \\
& =-\lambda \prod_{i=1}^{M-1}(\lambda-j)^{2} & & \text { if } N \text { is odd }
\end{aligned}
$$

Thus we see that

$$
\begin{gathered}
\lim _{\epsilon \rightarrow 0+} \lambda_{1}(\epsilon)=0, \quad \lim _{\epsilon \rightarrow 0+} \lambda_{2}(\epsilon)=1, \\
\lim _{\epsilon \rightarrow 0+} \lambda_{N}(\epsilon)=\left(\frac{N-1}{2}\right),
\end{gathered}
$$

so that the difference matrix is becoming ill-conditioned as $\epsilon \rightarrow 0$. For small values of $\epsilon$, we therefore cannot hope to use these simple direct methods, and we will discuss some special techniques for this problem in the next section.

It is interesting to note that we can actually find the rate at which $\lambda_{1}(\epsilon)$ is converging to 0 . Following a suggestion of Professor Ben Noble [10], we write

$$
\sum_{i=1}^{N} \frac{1}{\lambda_{i}}=\sum_{i=1}^{N}\left(A^{-1}\right)_{i i}
$$

Using the representation in Eq. (8), it can be shown that

$$
\left(A^{-1}\right)_{i i}=-\frac{\left(1+\sum_{k=1}^{i-1} \Delta_{k}\right)\left(\sum_{k=i}^{N} \Delta_{k}\right)}{c_{i} \Delta_{i}\left(1+\sum_{k=1}^{N} \Delta_{k}\right)}
$$


TABLB II

Smallest Eigenvalue $\lambda_{1}(\epsilon)$ of the Difference Matrix $A(\epsilon)(N=100)$

\begin{tabular}{cl||cc}
\hline$\epsilon$ & \multicolumn{1}{c||}{$\lambda_{1}(\epsilon)$} & $\epsilon$ & $\lambda_{1}(\epsilon)$ \\
\hline 0.100 & 0.58 & 0.0029 & $9.0 \times 10^{-13}$ \\
0.090 & 0.49 & 0.0028 & $4.3 \times 10^{-13}$ \\
0.080 & 0.40 & 0.0027 & $2.0 \times 10^{-13}$ \\
0.070 & 0.31 & 0.0026 & $8.7 \times 10^{-14}$ \\
0.060 & 0.23 & 0.0025 & $3.6 \times 10^{-14}$ \\
0.050 & 0.16 & 0.0024 & $1.4 \times 10^{-14}$ \\
0.040 & 0.090 & 0.0023 & $5.4 \times 10^{-15}$ \\
0.030 & 0.038 & 0.0022 & $1.9 \times 10^{-15}$ \\
0.020 & $7.1 \times 10^{-3}$ & 0.0021 & $6.1 \times 10^{-16}$ \\
0.010 & $6.2 \times 10^{-5}$ & 0.0020 & $1.8 \times 10^{-16}$ \\
0.009 & $2.3 \times 10^{-5}$ & 0.0019 & $5.0 \times 10^{-17}$ \\
0.008 & $6.8 \times 10^{-6}$ & 0.0018 & $1.3 \times 10^{-17}$ \\
0.007 & $1.5 \times 10^{-6}$ & 0.0017 & $2.8 \times 10^{-18}$ \\
0.006 & $2.1 \times 10^{-7}$ & 0.0016 & $5.4 \times 10^{-19}$ \\
0.005 & $1.6 \times 10^{-8}$ & 0.0015 & $9.0 \times 10^{-20}$ \\
0.004 & $4.2 \times 10^{-10}$ & 0.0014 & $1.3 \times 10^{-20}$ \\
0.003 & $1.8 \times 10^{-12}$ & 0.0013 & $1.5 \times 10^{-21}$ \\
0.002 & $1.8 \times 10^{-16}$ & 0.0012 & $1.3 \times 10^{-22}$ \\
0.001 & $4.4 \times 10^{-25}$ & 0.0011 & $9.1 \times 10^{-24}$ \\
\hline
\end{tabular}

Equations (11) and (12) can then be used to show that

$$
\begin{aligned}
\lim _{\epsilon \rightarrow 0+} \epsilon^{-M} \lambda_{1}(\epsilon) & =\left(h^{M+1} \prod_{i=1}^{M-1}\left(\frac{1}{2}-x_{i}\right)\right)^{-1} \quad \text { if } N \text { is even, } \\
& =\left(h^{M+1} \prod_{i=0}^{M-1}\left(\frac{1}{2}-x_{i}\right)\right)^{-1} \quad \text { if } N \text { is odd, }
\end{aligned}
$$

where $M=[(N+1) / 2]$. The terms in the right-hand side of this equation grow very rapidly as $h$ decreases. For example, for the case $N=100$ which we previously considered, this result is

$$
\lim _{\epsilon \rightarrow 0+} \epsilon^{-50} \lambda_{1}(\epsilon)=5.5870 \times 10^{136}
$$

5. Special Methods. We now discuss three special methods that can be applied to solving Eq. (9). Two of these techniques can also be applied to more general problems (for example, when the turning point is at $t=\alpha \in(0,1)$ instead of at $t=\frac{1}{2}$ ). However, we will not pursue such generalizations in this paper.

Method 1. For a given $\epsilon>0$, we define a sequence $\epsilon_{1}>\epsilon_{2}>\cdots>\epsilon_{k}=\epsilon$. We choose $\epsilon_{1}$ so that $W\left(x_{i}, \epsilon_{1}\right)$ can be computed by a stable direct method, and then we solve for $W\left(x_{i}, \epsilon_{i+1}\right)$ by Gauss-Seidel iteration with initial guess $W\left(x_{i}, \epsilon_{i}\right)$. This method has proved to be satisfactory, although it is quite slow. The primary 
disadvantage is that, in general, one would ${ }_{i}$ have difficulty in choosing parameters $\epsilon_{i}(1 \leqq i \leqq k-1)$ that would ensure that this method of computation is stable. Method 2. We can solve the system directly from the representation given in Eq. (7),

$$
W\left(x_{i}\right)=v_{0}+\left(v_{1}-v_{0}\right)\left(1+\sum_{i=1}^{i-1} \Delta_{i}\right)\left(1+\sum_{i=1}^{N} \Delta_{i}\right)^{-1} \quad(1 \leqq j \leqq N),
$$

where $\Delta_{i}=\prod_{k=1}^{i}\left(a_{k} / c_{k}\right)$. The problem, of course, is that it may be difficult to calculate $\Delta_{i}$ for $1 \leqq i \leqq N$, but there are two ways of avoiding this difficulty:

(i) Use the symmetry of the problem. Using the definitions of $a_{i}, b_{i}$, and $c_{i}$, it is easy to show that the coefficients satisfy:

$N$ even, $M=N / 2$ :

$$
\begin{aligned}
a_{M+i} & =c_{M+1-i} & & (1 \leqq j \leqq M), \\
c_{M+i} & =a_{M+1-i} & & (1 \leqq j \leqq M), \\
\Delta_{M+i} & =\Delta_{M-i} & & (1 \leqq j \leqq M-1), \\
\Delta_{N} & =1 ; & &
\end{aligned}
$$

$N$ odd, $M=(N+1) / 2$ :

$$
\begin{aligned}
a_{M+i} & =c_{M-i} & & (1 \leqq j \leqq M-1), \\
c_{M+i} & =a_{M-i} & & (1 \leqq j \leqq M-1), \\
\Delta_{M+i} & =\Delta_{M-i-1} & & (1 \leqq j \leqq M-2), \\
\Delta_{N} & =1 . & &
\end{aligned}
$$

Using these relations, we see that it is necessary only to calculate $\Delta_{i}$ for $1 \leqq i \leqq M$. Since $0<\Delta_{i+1} \leqq \Delta_{i}$ for $1 \leqq i \leqq M-1$, these values can easily be computed.

(ii) Factor out the power of $\epsilon$ in $\Delta_{i}$. For example, consider the case of even $N$ in the above problem, and let $M=N / 2$. Then

$$
\begin{aligned}
& \Delta_{i}=\epsilon^{i}\left[\prod_{k=1}^{i}\left(\frac{a_{k}}{\epsilon c_{k}}\right)\right] \quad \text { if } 1 \leqq i \leqq M, \\
& =\epsilon^{N-i}\left[\prod_{k=1}^{M}\left(\frac{a_{k}}{\epsilon c_{k}}\right)\right]\left[\prod_{k=M+1}^{i}\left(\frac{\epsilon a_{k}}{c_{k}}\right)\right] \quad \text { if } M+1 \leqq i \leqq N .
\end{aligned}
$$

Since

$$
\frac{a_{k}}{\epsilon c_{k}}=\frac{1}{\epsilon+h\left(\frac{1}{2}-x_{k}\right)} \quad(1 \leqq k \leqq M)
$$

and

$$
\frac{\epsilon a_{k}}{c_{k}}=\epsilon-h\left(\frac{1}{2}-x_{k}\right) \quad(M+1 \leqq k \leqq N),
$$

it is easy to calculate $\Delta_{i}$ in this form. It is clear that this procedure can be adapted to the case of odd $N$, and also to problems of this form in which the turning point is not at $t=\frac{1}{2}$.

Method 3. In this particular problem, we can use the symmetry relations discussed above to find that: 
$N$ even, $M=N / 2$ :

$$
W\left(x_{M+i}\right)=v_{1}+v_{0}-W\left(x_{M+1-i}\right) \quad(1 \leqq j \leqq M) ;
$$

$N$ odd, $M=(N+1) / 2$ :

$$
W\left(x_{M+i}\right)=v_{1}+v_{0}-W\left(x_{M-j}\right) \quad(0 \leqq j \leqq M-1) .
$$

If $N$ is odd and $M=(N+1) / 2$, it follows that $W\left(x_{M}\right)=\frac{1}{2}\left(v_{0}+v_{1}\right)$. Thus Eq. (9) is equivalent to the smaller problem

$$
\begin{gathered}
\epsilon W_{x \dot{x}}\left(x_{j}\right)+\left(\frac{1}{2}-x_{i}\right) \frac{\partial W\left(x_{j}\right)}{\partial x}=0 \quad(1 \leqq j \leqq M-1), \\
W(0)=v_{0}, \quad W\left(\frac{1}{2}\right)=\frac{1}{2}\left(v_{0}+v_{1}\right) .
\end{gathered}
$$

The matrix corresponding to this system is not ill-conditioned and, since we have reduced the dimension of the linear system by a factor of $\frac{1}{2}$, the method is also very fast. However, it cannot be applied directly to the case of even $N$, and clearly does not generalize to nonsymmetric problems.

Los Alamos Scientific Laboratory

Los Alamos, New Mexico 87544

1. I. BABUŠKa, Numerical Stability in the Solution of the Tri-Diagonal Matrices, Report BN-609, The Institute for Fluid Dynamics and Applied Mathematics, University of Maryland, College Park, Md., 1969.

2. I. BABUšKa, Numerical Stability in Problems in Linear Algebra, Report BN-663, The Institute for Fluid Dynamics and Applied Mathematics, University of Maryland, College Park, Md., 1970.

3. F. W. DorR, The Asymptotic Behavior and Numerical Solution of Singular Perturbation Problems with Turning Points, Ph.D. Thesis, University of Wisconsin, Madison, Wis., 1969.

4. F. W. DorR, "The numerical solution of singular perturbations of boundary value problems," SIAM J. Numer. Anal., v. 7, 1970, pp. 281-313.

5. F. W. DORR \& S. V. PARTER, "Singular perturbations of nonlinear boundary value problems with turning points," J. Math. Anal. Appl., v. 29, 1970, pp. 273-293.

6. F. W. DORR \& S. V. PARTER, Extensions of Some Results on Singular Perturbation Problems With Turning Points, Report LA-4290-MS, Los Alamos Scientific Laboratory, Los Alamos, N. M., 1969.

7. G. H. Golub, Matrix Decompositions and Statistical Calculations, Report CS-124, Computer Science Department, Stanford University, Stanford, Calif., 1969.

8. D. GreENSPAN, Numerical Studies of Two Dimensional, Steady State Navier-Stokes Equations for Arbitrary Reynolds Number, Report 9, Computer Sciences Department, University of Wisconsin, Madison, Wis., 1967.

9. W. D. MURPHY, "Numerical analysis of boundary-layer problems in ordinary differential equations," Math. Comp., v. 21, 1967, pp. 583-596. MR 37 \#1089.

10. B. Noble, Personal Communication, University of Wisconsin, Madison, Wis., Feb. $19,1970$.

11. C. E. PEARSON, “On a differential equation of boundary layer type," J. Mathematical Phys., v. 47, 1968, pp. 134-154. MR 37 \#3773.

12. C. E. PEARSON, "On non-linear ordinary differential equations of boundary layer type," J. Mathematical Phys., v. 47, 1968, pp. 351-358. MR 38 \#5400.

13. H. S. PRICE, R. S. VARGA \& J. E. WARREN, "Application of oscillation matrices to diffusion-convection equations," J. Mathematical Phys., v. 45, 1966, pp. 301-311. MR 34 \#7046.

14. H. S. PrICE \& R. S. VARGA, Error Bounds for Semidiscrete Galerkin Approximations of Parabolic Problems With Applications to Petroleum Reservoir Mechanics, SIAM-AMS Proc., vol. 2, Amer. Math. Soc., Providence, R. I., 1970, pp. 74-94.

15. R. S. VARGA, Matrix Iterative Analysis, Prentice-Hall, Englewood Cliffs, N. J., 1962. MR 28 \#1725.

16. J. H. Wriknson, The Algebraic Eigenvalue Problem, Clarendon Press, Oxford, 1965. MR 32 \#1894. 